\title{
Pathogenesis of the intravertebral vacuum of Kümmell's disease (Review)
}

\author{
DENGWEI HE ${ }^{1,2}$, WEIYANG YU ${ }^{2}$, ZHENZHONG CHEN ${ }^{2}$, LIANGCHEN LI $^{2}$, \\ KEJUN ZHU ${ }^{2}$ and SHUNWU FAN ${ }^{1}$ \\ ${ }^{1}$ Department of Orthopaedics, Sir Run Run Shaw Hospital, School of Medicine, Zhejiang University, Hangzhou, \\ Zhejiang 310016; ${ }^{2}$ Department of Orthopedics, Lishui Central Hospital, Wenzhou Medical University, \\ Lishui, Zhejiang 323000, P.R. China
}

Received February 15, 2016; Accepted May 19, 2016

DOI: $10.3892 /$ etm.2016.3369

\begin{abstract}
In this review, we explored the progress of the pathogenesis of Kümmell's disease intravertebral vacuum. Using different expressions of the same disease including 'Kümmell's disease', 'avascular necrosis after vertebral compression fracture (VCF)', 'post-traumatic vertebral osteonecrosis', 'vertebral pseudarthrosis', 'intravertebral vacuum (cleft or gas)', 'delayed vertebral collapse', 'VCF nonunion', and by conducting a search of the PubMed database, we analyzed the results to examine the pathogenesis of the intravertebral vacuum of Kümmell's disease after referring to pertinent literature on intravertebral vacuum of ischemic necrosis after VCF, and exploring the progress of pathogenesis of this disease. A number of discrepancies were identified within the pathogenesis of the intravertebral vacuum after VCF. There were statements such as avascular necrosis of the vertebral body, bone biomechanics, gas forming and other types of claims, all of which obtained clinical and biomechanical supporting evidence. Collectively, most of the researchers believe that Kümmell vertebral fracture syndrome was the comprehensive effect of multiple factors including osteoporosis, avascular necrosis of the vertebral body, and biomechanical changes following fracture. However, there are a number of discrepancies to be resolved and future studies are therefore needed.
\end{abstract}

\section{Contents}

1. Introduction

2. Causes of vertebral avascular necrosis

Correspondence to: Professor Shunwu Fan, Department of Orthopaedics, Sir Run Run Shaw Hospital, School of Medicine, Zhejiang University, 3 East Qingchun Road, Hangzhou, Zhejiang 310016, P.R. China

E-mail: shuwu_fan1@163.com

Key words: compression fractures, Kümmell's disease, osteoporotic vertebral vertebral body, intravertebral vacuum, pathogenesis
3. Air formation in vertebral body intravertebral vacuum

4. Avascular necrosis of vertebral body

5. Changes of bone biomechanics

6. Formation of vertebral pseudarthrosis

7. Other reasons

\section{Introduction}

Kümmell's disease is also known as avascular necrosis after osteoporotic vertebral compression fracture (OVCF) and was first reported by Herman Kümmell in 1895, after which there were few detailed studies. Other terms used to describe this disease include avascular necrosis after VCF, post-traumatic vertebral osteonecrosis, vertebral pseudarthrosis, intravertebral vacuum, cleft or gas, delayed vertebral collapse, and VCF nonunion (1).

In the recent two or three decades, with the development of computed tomography (CT) scan, magnetic resonance imaging (MRI) and other imaging examination methods, there is a rapid increasing trend of reported cases of this disease. According to the literature, the incidence of this disease in OVCF cases in elderly individuals accounts for 7-37\% (1). With the development of the aging population, the incidence has increased and has largely reduced the quality of life and expected lifespan of patients (2).

The intravertebral vacuum of Kümmell's disease usually occurs after osteoporotic VCFs of the middle-aged and elderly, which suggests that its occurrence was associated with osteoporosis. The pathogenesis of this disease may lie in tiny fractures occurring due to osteoporosis. Additionally, small blood-supplying artery in vertebral body is damaged due to tiny fractures and the vertebral blood supply is affected, leading to both parties interacting with each other and causing more serious blood vessel injuries. Thus osteoporosis is always regarded as one of the pathogenesis of vertebral avascular necrosis (3). In addition, previous findings showed that bone density was negatively correlated with the occurrence rate of intravertebral vacuum (2). From a pathological perspective, the decrease of osteoblasts of patients with osteoporosis was also considered as one of the risk factors of increasing vertebral ischemia incidence. 
In recent years, the particular 'intravertebral vacuum' of this disease has led to increased and investigations. The majority of scholars hold the view that osteoporosis was merely the basis of this disease and other factors of equal importance should be considered. Studies were performed with clinical imaging, on pathological and other aspects of Kümmell's disease and much valuable information was obtained (1-3). However, there are many factors leading to the occurrence of intravertebral vacuum of Kümmell's disease, thus its pathogenesis remains to be determined, and many discrepancies exist (4).

The present study was based on recent study results worldwide, especially the most popular imaging studies, which have drawn conclusions, and provide assistance for the clinical treatment strategies of this disease.

\section{Causes of vertebral avascular necrosis}

The majority of case studies support this argument and following an analysis of patients' disease history, the causes of bone avascular necrosis can be found in most of the patients, including long-term glucocorticoid treatment history, vein hardening, diabetes, alcoholism, pancreatitis, radiation therapy, acute trauma, tumor, and infection (1-3). Vertebral artery becomes narrow and the blood supply of the vertebral body is reduced due to atherosclerosis, fat embolism or degeneration. Benedek et al (5) described the two sides of Kummell disease. Firstly, after the initial injuries, the vertebra was not fully healed, or after healing was completed, the affected vertebra remained weakened. Secondly, the reparative process may involve the impairment of the blood supply, reaction to intra-osseous hemorrhage, and herniation of nucleus pilposus into the vertebral body. Repeated minor external forces lead to further progress in minor fractures, and ultimately lead to vertebral collapse fracture. Animal experiments by Drescher et al suggested that if methylprednisolone was used in pig vertebral body, the reduction of blood supply in loose bones of C6 and L6 vertebral body and the bottom of end plate leads to the small avascular necrosis of trabecular bone (6). This results in the phenomenon formation of similar vertebral body intravertebral vacuum, thus glucocorticoid is regarded as a crucial induciton factor of vertebral body bone nonunion and vertebral body intravertebral vacuum.

\section{Air formation in vertebral body intravertebral vacuum}

As for iconography, the vertebral body intravertebral vacuum of X-ray film usually appears as horizontal linear low-density air shadow, a small amount appears as quasi-circular shadow and located at the center of vertebral body or the side closer to compression vertebral body end plate. Occasionally it changes significantly, which means the air shadow expands when staying at extension position or dragging vertebral body, while it shrinks or even disappears at a fixed position (7). During the process of operating $\mathrm{CT}$, the diagnostic rate of intravertebral vacuum was slightly higher than that of X-ray as patients are mostly in the supine position, which appears as the irregular air shadow on cross section, but there is no essential difference from X-ray film (8). Considering the imaging diagnosis, CT scan may not be regarded as reliable evidence for the diagnosis and identification of Kümmell's disease. MRI has been widely applied in diagnosing Kümmell disease. Generally speaking, intravertebral vacuum on MRI appears as horizontal ribbon-like non-signal area on T1- or T2-weighted images (9). However, it may appear as different signals according to the difference of IVC exudate. When the exudate was gas, it appeared as a low signal on T1- and T2-weighted images. When the exudate was liquid, it appeared as low or high signal on T1- and T2-weighted images, respectively. Yu et al showed that vertebral fractures may have a vacuum effect, liquid phenomenon or be gas-liquid (10). The three imaging processes vary with regard to posture and length of bedrest time change. It means that liquid enters into the fracture gradually and replaces gas while the patient is in supine position.

Besides vertebral body, fracture sign also appears in intervertebral disks and other tissues. In recent years, the connection between fracture sign of vertebral body and that of intervertebral disks has been shown. Lafforgue et al reported that vertebral body with fracture sign is often accompanied with fracture sign of adjacent intervertebral disks, while vertebral body without fracture sign is rarely accompanied with fracture sign of adjacent intervertebral disks, while gas of part of vertebral body and intervertebral disks communicate with each other via endplate of fracture (8). Thus, the authors believed that fracture sign of intervertebral disks is formed because gas of intervertebral space passing the endplate fracture and diffusing to vertebral cavity. Karasick and Eason reported that gas cavity of fracture sign usually occurs in intervertebral disks and pseudo-articular surface and it was considered that gas can exacerbate the defect formation of vertebral endplate (11).

Besides gas formation and migratory routes, Armingeat et al analyzed gas composition (9). It was evaluated that there was $90-92 \%$ nitrogen in vertebral body according to prospective iconography, which was in accordance with gas composition from adjacent intervertebral disks.

\section{Avascular necrosis of vertebral body}

It is known that ischemic necrosis of bone often occurs in bone tissues that have mainly a single blood supply from terminal artery and is easier to cause ischemia after suffering injury and other factors, such as humerus and caput femoris. In anatomy, blood vessels of thoracic and lumbar vertebral body are derived from rami centrales of bigeminal segmental artery. The rear of two adjacent vertebral bodies were supplied by its rami posterior while anterior $1 / 3$ of it were supplied by single blood supply from the terminal artery and collateral branch was used for recycling, which may increase the risk of insufficiency of the anterior $1 / 3$ of blood vessels of the vertebral body. Interruption of blood supply and insufficiency of neovascularization of bone marrow are the likely causes of avascular necrosis of the vertebral body (12). The results were in concordance with those regarding vertebral collapse of Kümmell's disease, which often occurs in the anterior $1 / 3$ of vertebral body clinically (13). In addition, the increase of marrow cavity pressure caused by enterorrhagia may lead to a proportionate decrease in adipocyte size and blood flow of bone, which is also one of the factors that cause avascular necrosis of the vertebral body (14). In the study by Miller and Virkus, patients received spine blood vessel mammography which confirmed 
that reduction, shortage, or even closure of blood supply of vertebral body indicated that vertebral collapse is caused by insufficiency of vertebral blood supply based on part of vertebral collapse of vertebral fracture (15).

In pathology, the pathological manifestations of Kümmell's disease vertebral fracture signs are mainly osteonecrosis. In the study by Libicher et al 180 patients underwent vertebroplasty iconography and pathological biopsy (16). The results showed that of 12 patients diagnosed with osteonecrosis, 11 cases had vertebral fracture signs. Thus, CT manifestations of vertebral fracture sign are associated with pathology, suggesting that fracture sign can be regarded as a symptom of partial vertebral osteonecrosis and nonunion (rate of susceptibility was $85 \%$, specificity was $99 \%$, and positive predictive value was $91 \%$ ). Lin et al reported recently that MRI fluid sign can predict reliably osteonecrosis caused by vertebral tiny fracture (rate of susceptibility was $86 \%$, specificity was $100 \%$, and diagnostic odds ratios was 65) (17).

However, not all investigators support the mechanism. Ma et al and Fabbriciani et al reported that patients with vertebral collapse and fracture sign have several cardiovascular diseases or arteriosclerosis besides osteoporosis. Nevertheless, signs of osteonecrosis could not be found $(18,19)$.

\section{Changes of bone biomechanics}

In clinical manifestation, osteoporotic vertebral fracture with or without fracture sign often occurs in the junction of thoracolumbar vertebra. Consequently, it may be hypothesized that the decrease of shear stress causes vertebral tiny fracture and is the main reason for this disease. This is in accordance with a wider image of junction of thoracolumbar vertebra and a heavy load of the principle of biomechanics $(20,21)$. It is believed that vertebra after fracture is in a compression state with loss of vertebral height and kyphosis (22). Kyphosis moves barycenter of weighted backbone forward, changes the biomechanics environment of vertebra and leads to more severe fracture.

\section{Formation of vertebral peudarthrosis}

Vertebra has tiny fractures under external pressure. If the primary disease exists with negative bone balance, the tiny bone cannot usually recover and may cause fracture nonunion and exit movement activity of pseudarthrosis. It may lead to vertebral artery lesions and other secondary pathological changes. During the process of vertebral bone injury and restoration, mutual effects and mutual interactions potentially lead to the formation of a fracture sign (22). The study by Kim et al showed fracture influenced by vertebral osteoporotic bifid spine formed by tissue and pseudarthrosis of fibrocartilage, which is in accordance with movement of pseudarthrosis (23).

In histopathology, Libicher et al investigated the correlation of fracture sign CT scan after VCF and histology (16). The results of that study suggested that vertebral fracture sign can be a symptom of partial vertebral ischemic bone nonunion and the formation of pseudarthrosis (rate of susceptibility was $85 \%$, specificity was $99 \%$, and the positive predictive value was $91 \%)$.

\section{Other reasons}

Previous reports mentioned that the pathogenesis of Kümmell's disease may include bio-infection, bone fatigue caused by over-activity, formation of minimum hematoma, neurotrophic damage, fat embolism and other factors. Figura et al reported that the Helicobacter pylori infection rate in the experimental group was higher than that in the control group (24). However, it was not statistically significant. Thus, it can be seen as a potential risk factors A clinical study by Asaoka et al also supported this opinion and they believed that Helicobacter pylori infection is a risk factor of osteoporosis (25).

Theodorou mentioned that gas of vertebra with vertebral osteomyelitis may come from aerogenes (22). However, the pattern and distribution of gas were different from previous vertebrae necrosis under the circumstances, and gas produced by it was better equipped with the feature of soft tissue infection.

In conclusion, the findings on the formation mechanism of Kümmell's disease vertebral fracture sign in recent years have shown that it is the result of osteoporosis, vertebral ischemic necrosis, changes of biomechanics after fracture and various factors which are functioning coordinately. Hypotheses raised by researchers also obtained corresponding supporting evidence. According to these research results, clinicians can achieve more accurate analyses in the clinic by combining with actual cases to obtain better treatment strategies for the disease. Thus, most research on Kümmell's disease vertebral fracture sign was focused on iconography, pathology and conclusions of clinical features. More prospective clinical studies and biomechanics studies are required to verify results.

\section{Acknowledgements}

The present study was supported by the Project of the Ministry of Health Science and Technology of China (W2013ZT183), the Zhejiang Medical Research Foundation of China (nos. 2014-KYB315 and 2016KYA200) and the Zhejiang Science and Technology Program Foundation of China (2016C33245).

\section{References}

1. Lee SH, Kim ES and Eoh W: Cement augmented anterior reconstruction with short posterior instrumentation: a less invasive surgical option for Kummell's disease with cord compression. J Clin Neurosci 18: 509-514, 2011.

2. Wu AM, Chi YL and Ni WF: Vertebral compression fracture with intravertebral vacuum cleft sign: pathogenesis, image, and surgical intervention. Asian Spine J 7: 148-155, 2013.

3. Ito Y, Hasegawa Y, Toda K and Nakahara S: Pathogenesis and diagnosis of delayed vertebral collapse resulting from osteoporotic spinal fracture. Spine J 2: 101-106, 2002.

4. Yang H, Pan J and Wang G: A review of osteoporotic vertebral fracture nonunion management. Spine 39: B4-B6, 2014.

5. Benedek TG and Nicholas JJ: Delayed traumatic vertebral body compression fracture; Part II: Pathologic features. Semin Arthritis Rheum 10: 271-277, 1981.

6. Drescher W, Li H, Qvesel D, Jensen SD, Flo C, Hansen ES and Bünger C: Vertebral blood flow and bone mineral density during long-term corticosteroid treatment: an experimental study in immature pigs. Spine 25: 3021-3025, 2000.

7. Zhang K, Duan H, Xiang Z and Tu C: Surgical technique and clinical results for scapular allograft reconstruction following resection of scapular tumors. J Exp Clin Cancer Res 28: 45-55, 2009. 
8. Lafforgue P, Chagnaud C, Daumen-Legré V, Daver L, Kasbarian M and Acquaviva PC: The intravertebral vacuum phenomenon ('vertebral osteonecrosis'). Migration of intradiscal gas in a fractured vertebral body? Spine 22: 1885-1891, 1997.

9. Armingeat T, Pham T, Legre V and Lafforgue P: Coexistence of intravertebral vacuum and intradiscal vacuum. Joint Bone Spine 73: 428-432, 2006.

10. Yu CW, Hsu CY, Shih TT, Chen BB and Fu CJ: Vertebral osteonecrosis: MR imaging findings and related changes on adjacent levels. AJNR Am J Neuroradiol 28: 42-47, 2007.

11. Karasick D and Eason MA: Vertebral pneumatocyst mimicking susceptibility artifact on MR imaging. AJR Am J Roentgenol 170 221, 1998.

12. Freedman BA and Heller JG: Kummel disease: a not-so-rare complication of osteoporotic vertebral compression fractures. J Am Board Fam Med 22: 75-78, 2009.

13. Afzali B,Lechler RI and Hernandez-Fuentes MP: Allorecognition and the alloresponse: clinical implications. Tissue Antigens 69: 545-556, 2007

14. Saito S, Inoue A and Ono K: Intramedullary haemorrhage as a possible cause of avascular necrosis of the femoral head. The histology of 16 femoral heads at the silent stage. J Bone Joint Surg Br 69: 346-351, 1987.

15. Miller BJ and Virkus WW: Intercalary allograft reconstructions using a compressible intramedullary nail: a preliminary report. Clin Orthop Relat Res 468: 2507-2513, 2010.

16. Libicher M, Appelt A, Berger I, Baier M, Meeder PJ, Grafe I Dafonseca K, Nöldge G and Kasperk C: The intravertebral vacuum phenomen as specific sign of osteonecrosis in vertebral compression fractures: results from a radiological and histological study. Eur Radiol 17: 2248-2252, 2007.
17. Lin CL, Lin RM, Huang KY, Yan JJ and Yan YS: MRI fluid sign is reliable in correlation with osteonecrosis after vertebral fractures: a histopathologic study. Eur Spine J 22: 1617-1623, 2013.

18. Ma R, Chow R and Shen FH: Kummell's disease: delayed post-traumatic osteonecrosis of the vertebral body. Eur Spine J 19: 1065-1070, 2010

19. Fabbriciani G, Pirro M, Floridi P, Callarelli L, Manfredelli MR, Scarponi AM and Mannarino E: Osteoanabolic therapy: a non-surgical option of treatment for Kümmell's disease? Rheumatol Int 32: 1371-1374, 2012.

20. Li H, Liang CZ, Shen CC and Chen QX: Decreases in fluid shear stress due to microcracks: a possible primary pathogenesis of Kümmell's disease. Med Hypotheses 77: 897-899, 2011.

21. Pesce V, Piazzolla A, Moretti L, Carlucci S, Parato C, Maxy P and Moretti B: The vertebral biomechanic previous and after kyphoplasty. Aging Clin Exp Res 25 (Suppl 1): S71-S74, 2013.

22. Theodorou DJ: The intravertebral vacuum cleft sign. Radiology 221: 787-788, 2001.

23. Kim HS, Kim SH, Ju CI, Kim SW, Lee SM and Shin H: The role of bone cement augmentation in the treatment of chronic symptomatic osteoporotic compression fracture. J Korean Neurosurg Soc 48: 490-495, 2010.

24. Figura N, Gennari L, Merlotti D, Lenzi C, Campagna S, Franci B, Lucani B, Trabalzini L, Bianciardi L, Gonnelli C, et al: Prevalence of Helicobacter pylori infection in male patients with osteoporosis and controls. Dig Dis Sci 50: 847-852, 2005

25. Asaoka D, Nagahara A, Hojo M, Sasaki H, Shimada Y, Yoshizawa T, Osada T and Watanabe S: The relationship between $H$. pylori infection and osteoporosis in Japan. Gastroenterol Res Pract 2014: 340765, 2014. 